www.nature.com/jhg

\title{
SNPs on chromosome 5p15.3 associated with myocardial infarction in Japanese population
}

\author{
Asako Aoki ${ }^{1,2}$, Kouichi Ozaki ${ }^{1}$, Hiroshi Sato ${ }^{3}$, Atsushi Takahashi ${ }^{4}$, Michiaki Kubo ${ }^{5}$, Yasuhiko Sakata ${ }^{6}$, \\ Yoshihiro Onouchi ${ }^{1}$, Takahisa Kawaguchi ${ }^{7}$, Tsung-Hsien Lin $^{8,9}$, Hitoshi Takano ${ }^{2}$, Masahiro Yasutake ${ }^{2}$, \\ Po-Chao Hsu ${ }^{8}$, Shiro Ikegawa ${ }^{10}$, Naoyuki Kamatani ${ }^{4}$, Tatsuhiko Tsunoda ${ }^{7}$, Suh-Hang H Juo ${ }^{11,12,13}$, \\ Masatsugu Hori $^{6}$, Issei Komuro ${ }^{6}$, Kyoichi Mizuno ${ }^{2}$, Yusuke Nakamura ${ }^{14}$ and Toshihiro Tanaka ${ }^{1}$
}

Myocardial infarction (MI) occurs as the result of complex interactions of multiple genetic and environmental factors. By conducting a genome wide association study in a Japanese population using 210785 single nucleotide polymorphism (SNP) markers, we identified a novel susceptible locus for MI on chromosome 5p15.3. An SNP (rs11748327) in this locus showed significant association in several independent cohorts (combined $P=5.3 \times 10^{-13}$, odds ratio $=0.80$, comparison of allele frequency). Association study using tag SNPs in the same linkage disequilibrium block revealed that two additional SNPs (rs490556 and rs521660) conferred risk of MI. These findings indicate that the SNPs on chromosome 5p15.3 are novel protective genetic factors against MI.

Journal of Human Genetics (2011) 56, 47-51; doi:10.1038/jhg.2010.141; published online 25 November 2010

Keywords: association; genome; myocardial infarction; SNP

\section{INTRODUCTION}

Myocardial infarction (MI) leads to principal cause of death in developed countries. MI is characterized by the rapid development of coronary thrombosis following atherosclerotic plaque instability, ${ }^{1}$ which leads to necrosis of myocardium and might result in sudden death. Despite the change in lifestyle and recent development of biomarkers, pharmacological intervention and percutaneous coronary intervention using drug eluting stents, the mortality is still high.

We started genome wide association studies (GWAS) of this disorder using nearly 90000 gene-based single nucleotide polymorphisms (SNPs) (http://snp.ims.u-tokyo.ac.jp/) ${ }^{2}$ by high-throughput multiplex-PCR invader assay system, ${ }^{3}$ and identified several genes conferring risk of MI including LTA. ${ }^{4-6}$ Although the roles of these susceptible genes in MI pathogenesis are under investigation, these findings showed the potent power of GWAS, which is hypothesis free, to identify unexpected anchors to further understand the disease. Through examining the LTA cascade by combination of biological and genetic analyses, we have identified additional MI susceptible genes. ${ }^{7-9}$
Genetic variants that confer susceptibility to MI have been indicated to be present on several chromosomal loci. ${ }^{10-17}$ These studies, however, were conducted in individuals from European decent. Therefore, we carried out a systematic GWAS using 210785 SNPs for MI in Japanese population. We report here identification of SNPs on chromosome 5 p15.3 as a novel protective genetic factor against MI. We also examined Taiwanese population to see its universality in another population.

\section{MATERIALS AND METHODS \\ DNA samples}

For the genome wide association study and subsequent second-stage screening, MI case and control subjects (mixed cases with other diseases including asthma, breast cancer, lung cancer, hyperthyroidism, osteoporosis, chronic obstructive pulmonary disease, pollinosis and atopic dermatitis) were obtained from the BioBank Japan project (http://biobankjp.org/). The characteristics of the third, fourth cohorts and the diagnosis of define MI has been described previously. ${ }^{8,9}$ For Taiwanese population, subjects were recruited from the Kaohsiung Medical University Hospital, Taiwan. ${ }^{9}$ All Taiwanese subjects are of Chinese decent. All

\footnotetext{
${ }^{1}$ Laboratory for Cardiovascular Disease, Center for Genomic Medicine, RIKEN, Yokohama, Japan; ${ }^{2}$ Division of Cardiology, Department of Medicine, Nippon Medical School, Tokyo, Japan; ${ }^{3}$ School of Human Welfare Studies, Kwansei Gakuin University, Nishinomiya, Japan; ${ }^{4}$ Laboratory for Statistical Analysis, Center for Genomic Medicine, RIKEN, Yokohama, Japan; ${ }^{5}$ Laboratory for Genotyping Development, Center for Genomic Medicine, RIKEN, Yokohama, Japan; ${ }^{6}$ Department of Cardiovascular Medicine, Osaka University Graduate School of Medicine, Suita, Japan; ${ }^{7}$ Laboratory for Medical Informatics, Center for Genomic Medicine, RIKEN, Yokohama, Japan; ${ }^{8}$ Division of Cardiology, Department of Internal Medicine, Kaohsiung Medical University Hospital, Kaohsiung, Taiwan; ${ }^{9}$ Department of Internal Medicine, Kaohsiung Medical University, Kaohsiung, Taiwan; ${ }^{10}$ Laboratory for Bone and Joint Disease, Center for Genomic Medicine, RIKEN, Tokyo, Japan; ${ }^{11}$ Department of Medical Research, Kaohsiung Medical University Hospital, Kaohsiung, Taiwan; ${ }^{12}$ Graduate Institute of Medical Genetics, Kaohsiung Medical University, Kaohsiung, Taiwan; ${ }^{13}$ Center of Excellence for Environmental Medicine, Kaohsiung Medical University, Kaohsiung, Taiwan and ${ }^{14}$ Center for Genomic Medicine, RIKEN, Yokohama, Japan

Correspondence: Dr T Tanaka, Laboratory for Cardiovascular Diseases, Center for Genomic Medicine, RIKEN, 1-7-22, Suehiro-cho, Tsurumi-ku, Yokohama, Kanagawa 230-0045, Japan. 
study subjects provided written informed consent to participation in this study, or if they were under 20 years old, their parents gave consent. Characteristics of the study subjects were summarized in Supplementary Table 1. The protocol was approved by the Ethical Committee at the Center for Genomic Medicine, The institute of physical and chemical research (RIKEN), Yokohama and of each participating institution and by the Internal Review Board of the Kaohsiung Medical University Hospital, Kaohsiung.

\section{SNP genotyping}

The genotyping methods for GWAS and second-stage screening were described previously. ${ }^{18}$ For third- and fourth-stage screening, we used multiplex-PCR invader assay described previously. ${ }^{3}$ In the Taiwanese population, the SNPs were genotyped using the TaqMan SNP genotyping assay (Applied Biosystems, Foster City, CA, USA).

\section{Statistical analysis}

Haplotype block and haplotype frequency were estimated using Haploview v4.0. ${ }^{19}$ We used this software to select tag SNPs with a pairwise tagging mode and applied a permutation test for haplotype analysis. We also applied haplotype analysis using the program THESIAS ${ }^{20}$ and conditional log-likelihood with Akaike information criterion (AIC): $\mathrm{AIC}=-2 \times($ the maximized value of the conditional log-likelihood) $+2 \times$ (the number of parameters). As the number of parameters, we used the number of alleles/haplotypes with frequencies $>0.01$ that were used for each model. In the logistic regression analysis of an SNP, we first applied a one degree of freedom (1 d.f.) likelihood ratio test to determine whether a 1-d.f. multiplicative allelic effects model of a 2-d.f. full genotype model was more appropriate. ${ }^{20}$ As we found no significant difference from the full genotype model $(P>0.05)$, we assumed a multiplicative allelic effects mode. Next, we performed a forward logistic regression analysis, where we started by assessing whether the most significant SNP was sufficient to model the association among the SNP set. For this, we used a 1-d.f. likelihood ratio test for adding each of the remaining SNPs to the model by assuming multiplicative allelic effects for the additional SNPs. Relationship between patients' clinical profile and genotype information were assessed by one-way analysis of variance and $\chi^{2}$-test.

\section{Northern blot analysis}

Human multiple-tissue northern blots I, II (Clontech, Palo Alto, CA, USA) or First choice northern blot (Ambion, Austin, TX, USA) 1, 2 were pre-hybridized and hybridized with $\alpha-\left[{ }^{32} \mathrm{P}\right]$-dCTP-labeled genomic fragments prepared by PCR using 106 primer pairs as probes (Primer pairs are listed in Supplementary Table 2). Washed membranes were exposed to bioimaging plate for $4-6 \mathrm{~h}$. We detected signal with bioimaging analyzer (FLA7000, FUJIFILM, Tokyo, Japan) according to the manufacturer's instructions.

\section{RESULTS}

\section{Genome wide association analysis}

We performed staged GWAS that include three screening stages as shown in Figure 1. To avoid false-negative results, we set a very loose threshold in the first-stage screening. We first genotyped 268068 SNPs with $194 \mathrm{MI}$ cases and 1539 controls (first set of each MI and control) enrolled in BioBank Japan. We successfully obtained genotype information at 210785 SNP loci. The genomic inflation factor $(\lambda)$ was 1.03 on the basis of the P-values from the Cochran-Armitage trend test, indicating there is no population stratification. We then selected 8740 SNPs showing $P$-values $<0.02$ for the second-stage screening, and genotyped the second set of 1394 MI patients and 1425 control individuals (Supplementary Table 3). Distribution of $P$-values for these SNPs were summarized in Supplementary Table 4. We also assessed population stratification in second-stage samples by comparing with HapMap samples using principal component analyses, ${ }^{21}$ and found that these samples did not show any sign of population stratification (Supplementary Figure 1). After the second-stage screening, we identified two SNPs showing statistical significance after

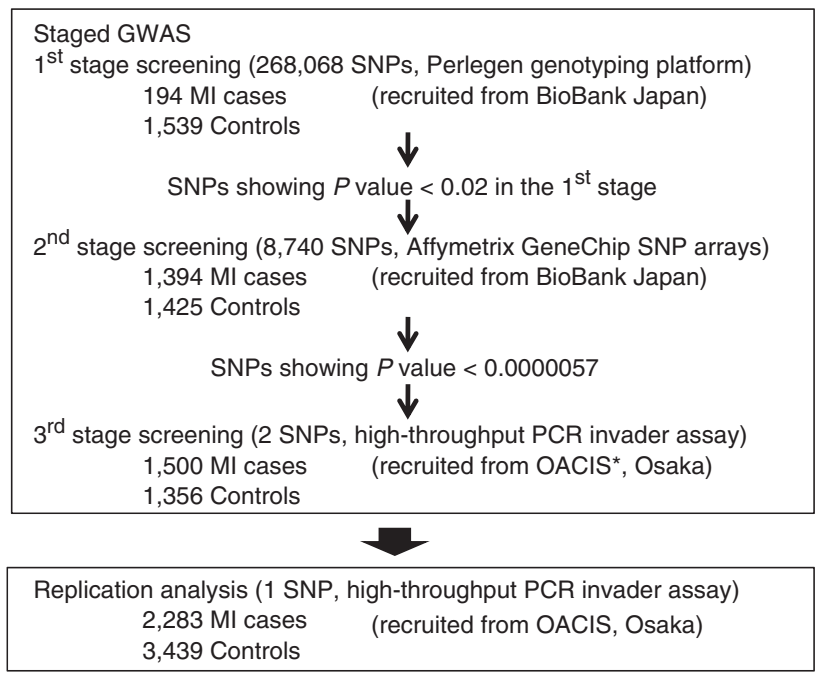

Figure 1 Study design for the GWAS. *Osaka Acute Coronary Insufficiency Study group.

Bonferroni's correction (cutoff $P$-value $<0.0000057$ ). One SNP (rs3782886) was located within BRAP on chromosome 12q24 (Supplementary Table 3), previously reported to be associated with susceptibility to MI in two Asian populations. ${ }^{9}$ The remaining SNP (rs11748327) on chromosome 5p15.3 showed $P$-value of $1.8 \times 10^{-6}$ in second-stage screening, and was verified by genotyping the third-stage samples (1500 cases and 1356 controls). Subsequent joint analyses for the associations of three panels showed the $P$-value with genome wide significance (combined $P=1.4 \times 10^{-9}$, odds ratio=0.77; Table 1 ). This association was further verified by replication panels with 2283 cases and 3439 controls (Table 1). Combined analysis of the four panels using Mantel-Haenszel test showed strong association of the SNP and MI, with a $\chi^{2}$ value of $56.0\left(P=5.3 \times 10^{-13}\right.$; comparison of allele frequency) and odds ratio was 0.80 (95\% confidence interval: 0.75-0.85; Table 1).

\section{Linkage disequilibrium and haplotype analysis}

The marker SNP (rs11748327) was located within $\sim 250 \mathrm{~kb}$ linkage disequilibrium (LD) block constructed on the basis of HapMap JPT data (http://www.hapmap.org) ${ }^{22}$ using Haploview software ${ }^{19}$ (Figure 2). To examine whether other genetic variation(s) in this block is associated with MI, we selected 15 tag SNPs in addition to rs11748327 from SNPs to have minor allele frequency $>5 \%$ with pairwise tagging and $r^{2}$ threshold of 0.8. We compared allelic frequency of these SNPs in the third panel of each MI and control and found that two additional SNPs (rs490556 and rs521660) were significantly associated with MI after Bonfferoni's correction (Table 2).

The two SNPs, rs490556 and rs521660, were in LD to the marker SNP rs11748327 with $r^{2}$ of 0.59 and 0.79 , respectively (Table 2). Then, we further genotyped the replication panel of 2283 cases and 3439 controls for the two SNPs and found again significant association between these SNPs and MI (Table 3). Two of the haplotypes based on these SNPs showed significant association with MI (Table 4). Therefore, we further examined the effect of these haplotypes by THESIAS ${ }^{20}$ and observed a significant effect on disease susceptibility between the most and second-most frequent haplotypes that could be distinguished by rs490556. Considering the conditional log-likelihoods with AIC, indicating that rs490556 revealed smaller AIC value than the haplotype model, we assumed that rs490556 alone rather than the 
Table 1 Association of rs11748327 SNP with MI

\begin{tabular}{|c|c|c|c|c|c|c|c|c|c|c|c|c|c|c|c|c|c|c|c|}
\hline \multirow[b]{2}{*}{ Stage } & \multirow[b]{2}{*}{ Panel } & \multicolumn{7}{|c|}{$M I$} & \multicolumn{7}{|c|}{ Controls } & \multicolumn{4}{|c|}{ Comparison of allele frequency } \\
\hline & & 11 & $\%$ & 12 & $\%$ & 22 & $\%$ & SUM & 11 & $\%$ & 12 & $\%$ & 22 & $\%$ & SUM & $\chi^{2}$ & P-value & $O R$ & $95 \% \mathrm{Cl}$ \\
\hline \multirow[t]{4}{*}{ Staged GWAS } & $1 \mathrm{st}$ & 130 & 67.0 & 52 & 26.8 & 12 & 6.2 & 194 & 866 & 56.3 & 575 & 37.4 & 97 & 6.3 & 1538 & 5.5 & $1.9 \times 10^{-2}$ & 0.73 & $0.56-0.95$ \\
\hline & 2 nd & 896 & 64.4 & 436 & 31.3 & 60 & 4.3 & 1392 & 758 & 55.3 & 531 & 38.7 & 82 & 6.0 & 1371 & 22.8 & $1.8 \times 10^{-6}$ & 0.74 & $0.83-0.65$ \\
\hline & $3 r d$ & 938 & 62.8 & 480 & 32.1 & 76 & 5.1 & 1494 & 770 & 57.1 & 490 & 36.4 & 88 & 6.5 & 1348 & 10.2 & $1.4 \times 10^{-3}$ & 0.82 & $0.72-0.93$ \\
\hline & Combined & & & & & & & & & & & & & & & 40.7 & $1.4 \times 10^{-9 *}$ & 0.77 & $0.71-0.84$ \\
\hline \multirow[t]{2}{*}{ Validation } & Replication & 1437 & 62.9 & 740 & 32.4 & 106 & 4.6 & 2283 & 1964 & 57.5 & 1254 & 36.7 & 197 & 5.8 & 3415 & 16.7 & $4.4 \times 10^{-5}$ & 0.83 & $0.76-0.91$ \\
\hline & Combined & & & & & & & & & & & & & & & 56.0 & $5.3 \times 10^{-13 *}$ & 0.80 & $0.75-0.85$ \\
\hline
\end{tabular}

Abbreviations: $\mathrm{Cl}$, confidence interval; GWAS, genome wide association studies; MI, myocardial infarction; OR, odds ratio; SNP, single nucleotide polymorphism.

*P-value was calculated by Mantel-Haenszel test.

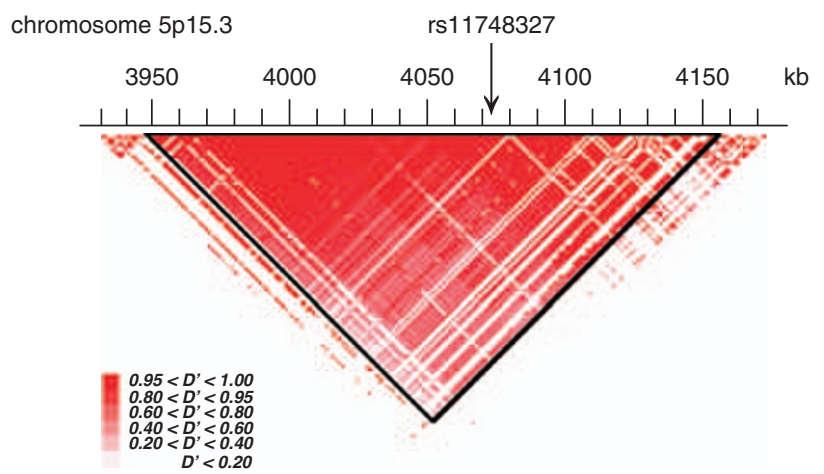

Figure 2 LD $\left(D^{\prime}\right)$ block containing the marker SNP (rs11748327) on chromosome 5 p15.3.

haplotypes well explained an association with MI. We also applied a logistic regression analysis to search for combinatorial effects of other SNPs to rs490556, but failed to find them. These results indicated that rs490556 itself or other SNPs with high LD to rs490556 are genetically associated with MI.

We also examined the possibility of confounding effect by age, sex and classical risk factors including diabetes, hypertension, smoking, hyperlipidemia within patients group using one-way analysis of variance and $\chi^{2}$ test, and found no relation between genotype and these factors (data not shown), indicating that the significant SNPs are an independent risk factor of MI.

We further conducted the association between the three tag SNPs and MI with $\sim 550$ cases and 800 controls from a Taiwanese population. However, the results were not consistent with the association for MI in Japanese population (Supplementary Table 5).

\section{Gene discovery at $5 \mathrm{p} 15.3$ locus}

National Center for Biotechnology Information database (http:// www.ncbi.nlm.nih.gov) contained only one expressed sequence tag, DA489076.1, in the genomic region within the LD block. Therefore, we examined expression of DA489076.1 in cDNA derived from 13 human tissues including heart, lung, liver, skeletal muscle, placenta, peripheral blood leukocyte, lymph node, adipose, aorta, brain, fetal brain, coronary artery smooth muscle and coronary artery endothelial cells. However, the expression was not detectable in all tissues examined (data not shown). To explore whether other unidentified transcripts are present in this genomic region, we examined mRNA expression in human adult tissues by northern blot analyses using 106 amplicons as probes (Primer pairs are listed in Supplementary Table 2) that cover the entire genomic region of the block except for repetitive sequences. We could not find obvious signal in all tissues examined (data not shown). Although we also examined micro-RNA (miRNA) and copy number variation databases (http://www.mirbase.org/search.shtml and https://gwas.lifesciencedb.jp/cgi-bin/cnvdb/cnv_top.cgi, respectively), we were not able to obtain any information for the genomic region.

\section{DISCUSSION}

Through a GWAS in a Japanese population using 210785 SNP markers, we identified SNPs on chromosome 5p15.3 as a novel protective genetic factor against MI. The association that we observed in the Japanese population could not be replicated in the Taiwanese population. This might be due to a lack of the power $(1-\beta ; 0.17$ in comparison of allele frequencies for these SNPs) or genetic difference between the two populations. We also cannot exclude the possibility that other variants in this genomic region confer risk of $\mathrm{MI}$ in the Taiwanese population. The loci on chromosome 5p15.3 and BRAP were not detected in the previous GWAS from Europe and the United States; this failure may be due to the difference among ethnicity in allelic frequencies, which affects power of the study and also the effect size. Other reasons might include the ethnic difference in the precise LD pattern, possibility of unidentified hidden SNPs for Caucasian decent, various biases such as publication bias, leaving open the question of association in other populations for these loci.

We could not find replicated previous results for LTA and PSMA6 in the first-stage screening. The estimated powers of the first-stage screening to replicate positive association for LTA and PSMA6 were 0.28 and 0.24 , respectively. The significant SNP in LGALS2 was not on the SNP list of Perlegen genotyping system. Therefore, we think one of the reasons might be lack of the power of this study. Biases including publication bias, sampling bias, cannot be excluded. For chromosome 9p21 locus, the $P$-value in the first-stage screening was 0.0018 (rs1412834). At the second stage, it was 0.0098 and did not pass the threshold. As our aim did not include replication of the previous findings, threshold $P$-value at each screening stage was not appropriate for replication study.

In the genomic region of the LD block on chromosome 5p15.3, we were not able to detect any transcript by our analyses. It is possible we cannot detect unidentified some small non-coding RNAs $<100$ base pairs, particularly miRNAs. miRNA has important functions in gene regulation in animals and plants by binding to target sites in the $3^{\prime}$ untranslated regions on mRNAs of protein-coding genes to direct their posttranscriptional repression. ${ }^{23}$ In fact, recent studies indicated that a single substitution in the mach of the miRNA seed to its target site can abolish gene repression. ${ }^{24}$ SNPs in miRNA including pri-miRNAs, pre-miRNAs and mature miRNA could influence the processing and/or target selection of miRNAs and affect 
Table 2 Association analysis of the 15 tag SNPs with MI

\begin{tabular}{|c|c|c|c|c|c|c|c|c|c|c|c|c|c|c|c|c|}
\hline \multirow[b]{2}{*}{$d b S N P I D$} & \multicolumn{7}{|c|}{$M I$} & \multicolumn{7}{|c|}{ Controls } & \multirow[b]{2}{*}{ P-value } & \multirow{2}{*}{$\begin{array}{c}r^{2} \text { with } \\
\text { rs11748327 }\end{array}$} \\
\hline & 11 & $\%$ & 12 & $\%$ & 22 & $\%$ & SUM & 11 & $\%$ & 12 & $\%$ & 22 & $\%$ & SUM & & \\
\hline rs $505800 A>G$ & 763 & 51.4 & 599 & 40.3 & 123 & 8.3 & 1485 & 666 & 49.6 & 551 & 41.1 & 125 & 9.3 & 1342 & $4.0 \times 10^{-1}$ & 0.3 \\
\hline rs2008927 C> T & 700 & 47.3 & 614 & 41.5 & 166 & 11.2 & 1480 & 650 & 49.2 & 524 & 39.6 & 148 & 11.2 & 1322 & $7.2 \times 10^{-1}$ & 0.18 \\
\hline rs631942 G>A & 795 & 53.9 & 558 & 37.8 & 122 & 8.3 & 1475 & 660 & 48.9 & 569 & 42.1 & 121 & 9.0 & 1350 & $2.9 \times 10^{-1}$ & 0.4 \\
\hline $\mathrm{rs} 521660 \mathrm{G}>\mathrm{A}$ & 762 & 55.3 & 515 & 37.3 & 102 & 7.4 & 1379 & 627 & 49.1 & 530 & 41.5 & 120 & 9.4 & 1277 & $1.5 \times 10^{-2}$ & 0.79 \\
\hline rs1187466 T>A & 630 & 44.3 & 630 & 44.3 & 162 & 11.4 & 1422 & 579 & 46.9 & 540 & 43.8 & 115 & 9.3 & 1234 & $1.1 \times 10^{-1}$ & 0.11 \\
\hline rs1187463 C >A & 743 & 50.4 & 600 & 40.7 & 130 & 8.8 & 1473 & 607 & 46.0 & 563 & 42.7 & 150 & 11.4 & 1320 & $7.5 \times 10^{-2}$ & 0.65 \\
\hline rs903083 C>T & 1334 & 89.4 & 156 & 10.5 & 2 & 0.1 & 1492 & 1180 & 88.0 & 155 & 11.6 & 6 & 0.4 & 1341 & $2.6 \times 10^{-1}$ & 0.02 \\
\hline rs10512709 G >A & 607 & 41.3 & 682 & 46.5 & 179 & 12.2 & 1468 & 559 & 42.3 & 611 & 46.3 & 151 & 11.4 & 1321 & $8.0 \times 10^{-1}$ & 0.17 \\
\hline rs1187477 C> T & 1169 & 77.9 & 308 & 20.5 & 23 & 1.5 & 1500 & 1025 & 75.6 & 311 & 22.9 & 20 & 1.5 & 1356 & $3.0 \times 10^{-1}$ & 0.04 \\
\hline
\end{tabular}

Abbreviations: MI, myocardial infarction; SNP, single nucleotide polymorphism.

${ }^{a}$ Comparison of allelic frequency and adjusted for Bonferroni's correction.

Table 3 Association of the rs490556 and rs521660 with MI

\begin{tabular}{|c|c|c|c|c|c|c|c|c|c|c|c|c|c|c|c|c|c|c|c|}
\hline$d b S N P I D$ & Samples & 11 & $\%$ & 12 & $\%$ & 22 & $\%$ & Total & 11 & $\%$ & 12 & $\%$ & 22 & $\%$ & Total & $\chi^{2}$ & P-value & $O R$ & $95 \% \mathrm{Cl}$ \\
\hline \multirow{2}{*}{ rs490556 T>C } & Replication & 1324 & 58.4 & 802 & 35.4 & 141 & 6.2 & 2267 & 1786 & 51.9 & 1399 & 40.68 & 254 & 7.4 & 3439 & 20.6 & $5.7 \times 10^{-6}$ & 0.82 & $0.75-0.89$ \\
\hline & Combined & & & & & & & & & & & & & & & 33.8 & $4.0 \times 10^{-9^{*}}$ & 0.81 & $0.76-0.87$ \\
\hline rs521660 G>A & $3 r d$ & 762 & 55.3 & 515 & 37.3 & 102 & 7.4 & 1379 & 627 & 49.1 & 530 & 41.504 & 120 & 9.4 & 1277 & 10.9 & $9.4 \times 10^{-4}$ & 0.82 & $0.72-0.92$ \\
\hline
\end{tabular}

Abbreviations: $\mathrm{Cl}$, confidence interval; MI, myocardial infarction; OR, odds ratio; SNP, single nucleotide polymorphism.

${ }^{*} P$-values were calculated by Mantel-Haenszel test.

Table 4 Haplotype analysis

\begin{tabular}{|c|c|c|c|c|c|c|c|}
\hline \multirow[b]{3}{*}{ Haplotype } & \multicolumn{3}{|c|}{$\begin{array}{l}\text { SNP IDS } \\
\text { Risk allele }\end{array}$} & \multicolumn{2}{|c|}{$\begin{array}{l}\text { Haplotype } \\
\text { frequency }\end{array}$} & \multicolumn{2}{|c|}{$\begin{array}{c}\text { Comparison of } \\
\text { haplotype frequency }\end{array}$} \\
\hline & rs490556 & rs11748327 & rs521660 & Case & Control & $\chi^{2}$ & P-value \\
\hline & $T$ & C & G & & & & \\
\hline Haplotype 1 & $\mathrm{~T}$ & C & G & 0.727 & 0.688 & 30.7 & $3.0 \times 10^{-8}$ \\
\hline Haplotype 2 & C & $\mathrm{T}$ & A & 0.176 & 0.213 & 37.1 & $1.1 \times 10^{-9}$ \\
\hline
\end{tabular}

Abbreviation: SNP, single nucleotide polymorphism.

miRNA-mediated translational suppression. ${ }^{25}$ Therefore, the SNPs on chromosome 5 p15.3 might be located within the region encoding unidentified miRNAs, affect their functions, and contribute to the development and/or progression of CAD. Although it is very difficult to reveal function of the SNP with the present knowledge, we think the increasing attention to function and/or higher-order structure of genome and subsequent progress will help to solve this problem.
We believe that knowledge of genetic factors contributing to its pathogenesis provides a useful clue for the development of diagnostic methods, treatments and preventive measures for this common but serious disorder.

\section{ACKNOWLEDGEMENTS}

We thank Maki Takahashi, Mayumi Yoshii, Saori Kawakami, Rumiko Oishi, Makiko Matsuda, Taeko Nakajima and Michiko Nakamura for their assistance. 
We also thank all the members of OACIS, the Rotary Club of Osaka-Midosuji District 2660 Rotary International and BioBank Japan for their contribution to the completion of our study. This work was conducted as a part of the BioBank Japan Project that was supported by the Ministry of Education, Culture, Sports, Sciences and Technology of the Japanese government. This work was also supported in part by grants from the Takeda science foundation, the Uehara science foundation, the Naito foundation, the Mitsubishi foundation, the Tokyo Biochemical Research foundation and NHRI-Ex96-9607PI (Taiwan).

1 Libby, P \& Theroux, P Pathophysiology of coronary artery disease. Circulation 111, 3481-3488 (2005).

2 Haga, H, Yamada, R, Ohnishi, Y, Nakamura, Y \& Tanaka, T Gene-based SNP discovery as part of the Japanese Millennium Genome project; identification of 190562 genetic variations in the human genome. J. Hum. Genet. 47, 605-610 (2002).

3 Ohnishi, Y, Tanaka, T, Ozaki, K, Yamada, R, Suzuki, H \& Nakamura, Y A high-throughput SNP typing system for genome-wide association studies. J. Hum. Genet. 46, 471-477 (2001).

4 Ozaki, K, Ohnishi, Y, lida, A, Sekine, A, Yamada, R, Tsunoda, T et al. Functional SNPs in the lymphotoxin- $\alpha$ gene that are associated with susceptibility to myocardial infarction. Nat. Genet. 32, 650-654 (2002).

5 Ishii, N, Ozaki, K, Sato, H, Mizuno, H, Saito, S, Takahashi, A et al. Identification of a novel non-coding RNA, MIAT, that confers risk of myocardial infarction. J. Hum. Genet. 51, 1087-1099 (2006).

6 Ebana, Y, Ozaki, K, Inoue, K, Sato, H, lida, A, Lwin, H et al. A functional SNP in ITIH3 is associated with susceptibility to myocardial infarction. J. Hum. Genet. 52, 220-229 (2007).

7 Ozaki, K, Inoue, K, Sato, H, lida, A, Ohnishi, Y, Sekine, A et al. Functional variation in LGALS2 confers risk of myocardial infarction and regulates lymphotoxin-a secretion in vitro. Nature 429, 72-75 (2004).

8 Ozaki, K, Sato, H, lida, A, Mizuno, H, Nakamura, T, Miyamoto, Y et al. A functional SNP in PSMA6 confers risk of myocardial infarction in the Japanese population. Nat. Genet. 38, 921-925 (2006).

9 Ozaki, K, Sato, H, Inoue, K, Tsunoda, T, Sakata, Y, Mizuno, $\mathrm{H}$ et al. SNPs in BRAP associated with risk of myocardial infarction in Asian populations. Nat. Genet. 41, 329-333 (2009).

10 Helgadottir, A, Thorleifsson, G, Manolescu, A, Gretarsdottir, S, Blondal, T, Jonasdottir, A et al. A common variant on chromosome $9 p 21$ affects the risk of myocardial infarction. Science 316, 1491-1493 (2007)
11 McPherson, R, Pertsemlidis, A, Kavaslar, N, Stewart, A, Roberts, R, Cox, D R et al. A common allele on chromosome 9 associated with coronary heart disease. Science 316, 1488-1491 (2007).

12 Samani, N J, Erdmann, J, Hall, A S, Hengstenberg, C, Mangino, M, Mayer, B, et al. WTCCC and the Cardiogenics Consortium Genome-wide association analysis of coronary artery disease. N. Engl. J. Med. 357, 443-453 (2007).

13 Wellcome Trust Case Control Consortium. Genome-wide association study of 14000 cases of seven common diseases and 3000 shared controls. Nature 447, 661-678 (2007).

14 Erdmann, J, Großhennig, A, Braund, P S, König, I R, Hengstenberg, C, Hall, A S, et al. Italian Atherosclerosis, Thrombosis, and Vascular Biology Working Group; Myocardial Infarction Genetics Consortium; Wellcome Trust Case Control Consortium; Cardiogenics Consortium New susceptibility locus for coronary artery disease on chromosome 3q22.3. Nat. Genet. 41, 280-282 (2009).

15 Gudbjartsson, D F, Bjornsdottir, U S, Halapi, E, Helgadottir, A, Sulem, P, Jonsdottir, G $\mathrm{M}$ et al. Sequence variants affecting eosinophil numbers associate with asthma and myocardial infarction. Nat. Genet. 41, 342-347 (2009).

16 Myocardial Infarction Genetics Consortium. Genome-wide association of early-onset myocardial infarction with single nucleotide polymorphisms and copy number variants. Nat. Genet. 41, 334-341 (2009).

17 Trégouët, D A, König, I R, Erdmann, J, Munteanu, A, Braund, P S, Hall, A S, et al. Wellcome Trust Case Control Consortium; Cardiogenics Consortium Genome-wide haplotype association study identifies the SLC22A3-LPAL2-LPA gene cluster as a risk locus for coronary artery disease. Nat. Genet. 41, 283-285 (2009).

18 Unoki, H, Takahashi, A, Kawaguchi, T, Hara, K, Horikoshi, M, Andersen, G et al. SNPs in KCNQ1 are associated with susceptibility to type 2 diabetes in East Asian and European populations. Nat. Genet. 40, 1098-1102 (2008).

19 Barrett, J C, Fry, B, Maller, J \& Daly, M J Haploview: analysis and visualization of LD and haplotype maps. Bioinformatics 21, 263-265 (2005).

20 Tregouet, D A \& Garelle, V A new JAVA interface implementation of THESIAS: testing haplotype effects in association studies. Bioinformatics 23, 1038-1039 (2007).

21 Price, A L, Patterson, N J, Plenge, R M, Weinblatt, M E, Shadick, N A \& Reich, D Principal components analysis corrects for stratification in genome-wide association studies. Nat. Genet. 38, 904-909 (2006).

22 The International HapMap Consortium. A haplotype map of the human genome. Nature 437, 1299-1320 (2005).

23 Rinn, J L, Kertesz, M, Wang, J K, Squazzo, S L, Xu, X, Brugmann, S A et al. Functional demarcation of active and silent chromatin domains in human $\mathrm{HOX}$ loci by noncoding RNAs. Cell 129, 1311-1323 (2007).

24 Brennecke, J, Stark, A, Russell, R B \& Cohen, S M Principles of microRNA-target recognition. PloS Biol. 3, e85 (2005).

25 Duan, R, Pak, C \& Jin, P Single nucleotide polymorphism associated with mature miR-125a alters the processing of pri-miRNA. Hum. Mol. Genet. 16, 1124 (2007).

Supplementary Information accompanies the paper on Journal of Human Genetics website (http://www.nature.com/jhg) 